\title{
Acompañamiento de estudiantes monitores y uso de software computarizado en Prácticas de Familiarización en Contaduría Pública y Finanzas. UNAN-Managua; FAREM-Estelí
}

\section{Accompaniment of assistant students and use of computerized software in Familiarization internship in Public Accounting and Finance. UNAN-Managua; FAREM-Estelí}

Jeyling María Alfaro Manzanares ${ }^{1}$

Recibido: 14 de noviembre de 2018, Aceptado: 15 de febrero de 2019

\section{RESUMEN}

En la actualidad durante el proceso de formación de los estudiantes principalmente en Contaduría Pública y Finanzas es necesario que ellos dominen los ambientes, módulos que constituyen un software contable, durante este semestre se propuso desde un inicio el uso del sistema, por lo que se cuenta la experiencia vivida, las dificultades y los logros alcanzado. En un grupo amplio como son los de Contaduría aproximadamente de 41, el apoyo de los estudiantes monitores, quienes fueron vital para el éxito de esta asignatura. Espero que al leer este ensayo puedan percibir la satisfacción que como docente se experimentó, obviamente cada vez será mejor, aplicando nuevas estrategias.

Palabras claves: contextualización; innovación; práctica docente; innovación pedagógica.

\section{ABSTRACT}

Nowadays during the process of training students, mainly in Public Accounting and Finance, it is necessary to manage the environments, and modules that set up an accounting software. During the first semester 2018, the use of this system was proposed, in addition in this essay, the lived experience, difficulties, and achievements are shared. We worked with a group of 41 students of accounting and had the support of assisting students, who were helpful for the success of this subject. I hope that when reading this essay you can perceive the satisfaction that as a teacher of UNAN-Managua, FAREMEstelí, it was experienced, clearly we will continue working with new strategies so that the learning development of students will improve.

Keywords: contextualization; innovation; teaching practice; pedagogical innovation.

1 Docente del departamento de Ciencias Económicas y Administrativas. UNAN-Managua/FAREM-Estelí. Correo electrónico: jeyfaman@yahoo.es

Copyright (c) 2018 Revista Multi-Ensayos.

(c) (1) (-) Este trabajo está licenciado bajo una Licencia Internacional Creative Commons 4.0 Atribución-NoComercial-Compartirlgual. 


\section{INTRODUCCIÓN}

LEl desarrollo de las prácticas en la formación de los futuros profesionales es de vital importancia. En la experiencia obtenida en este semestre donde se facilitó en conjunto con otro docente la asignatura de práctica de familiarización en donde se atendieron 41 estudiantes en conjunto fue bastante enriquecedor sin embargo al inició se experimentaban problemas en la asimilación de los contenidos.

Se inicia con la modalidad de esta asignatura en conjunto se elaboró un caso práctico donde el estudiante de manera individual debía registrar operaciones contables que se realizan en una empresa comercial, la variante en este año se planteó con el apoyo de estudiantes monitores y el uso de software computarizado llamado Mónica.

Es interesante el desarrollo de estas prácticas porque cada estudiante evidenciaba la debilidad en el uso de herramientas de office y uso del software, este último es importante que el profesional contable se vaya familiarizando dado que en el desarrollo de su ejercicio profesional es una herramienta muy necesaria para presentar de acuerdo a las exigencias del mercado.

\section{DESARROLLO}

Es importante abordar ciertos conceptos que son de utilidad para el desarrollo de este ensayo dentro de los cuales se puede mencionar:

MONICA es el programa de computador ideal para un negocio. Le permite realizar las facturas, controlar el inventario, realizar listas de precios, tener un archivo de clientes, proveedores, manejar las cuentas por cobrar, las cuentas corrientes, y la contabilidad básica de su empresa. Puede ser utilizado en cualquier país del habla castellana, tiene muchas facilidades con programas para el manejo contable como (Tecnotel, 2010):

Realiza facturación: Crea facturas, lleva el control de éstas, obtiene totales de ventas por clientes, por fechas, por ítem, por vendedor. Permite versatilidad en la impresión de facturas (forma pre-impresa, hoja simple/media pág.). Permite realizar estimados, así como la facilidad de imprimir las facturas y estimados en inglés y/o castellano. Puede realizar devoluciones. Cuando hace una factura el inventario automáticamente queda actualizado.

Inventario: Usted puede llevar el control de los ítems de su inventario: asignando códigos, conociendo en cualquier momento el precio, el costo, y la cantidad disponible de un determinado ítem. Así mismo tiene la capacidad de determinar el movimiento de ítems para un período determinado (Cuánto se vendió, montos, etc.). Determina el costo de su inventario. Lleva el Kardex de los productos.

Clientes: Usted puede determinar mediante el auxilio de MONICA cuales han sido sus ventas por clientes, cuáles son sus mejores clientes, cuanto les ha vendido y cuando Cuentas por cobrar (Cartera). Permite manejar las ventas al crédito que realiza con sus clientes: Vencimiento de facturas, Cargos financieros, puede obtener los estados de cuenta por cada cliente. Cierre de periodos que le permite determinar cuánto le debe cada uno de sus clientes.

Cuentas por pagar: Maneja las compras a sus suplidores, MONICA le permite realizar cotizaciones (Averiguación del precio de un producto) y luego realizar la orden de compra (Formalizar la compra del producto). Usted puede saber cuánto le debe a un abastecedor, si la compra fue al crédito, cuando se vence la factura, etc. 
Cheques: Lleva un control preciso de los montos girados, los cheques que han sido cobrados, aquellos que faltan ser cobrados, montos totales para un período. Asimismo, puede imprimir directamente los cheques con total seguridad (montos en números y en letras), utilizando formato preimpreso y numeración dada por computador. También puede obtener un reporte de reconciliación bancaria. Permite saber cuál es su saldo. Se puede tener desde una o más cuentas corrientes. La impresión de cheques puede ser en Castellano/inglés.

La siguiente imagen es un ejemplo de una Empresa que usa el sistema MONICA

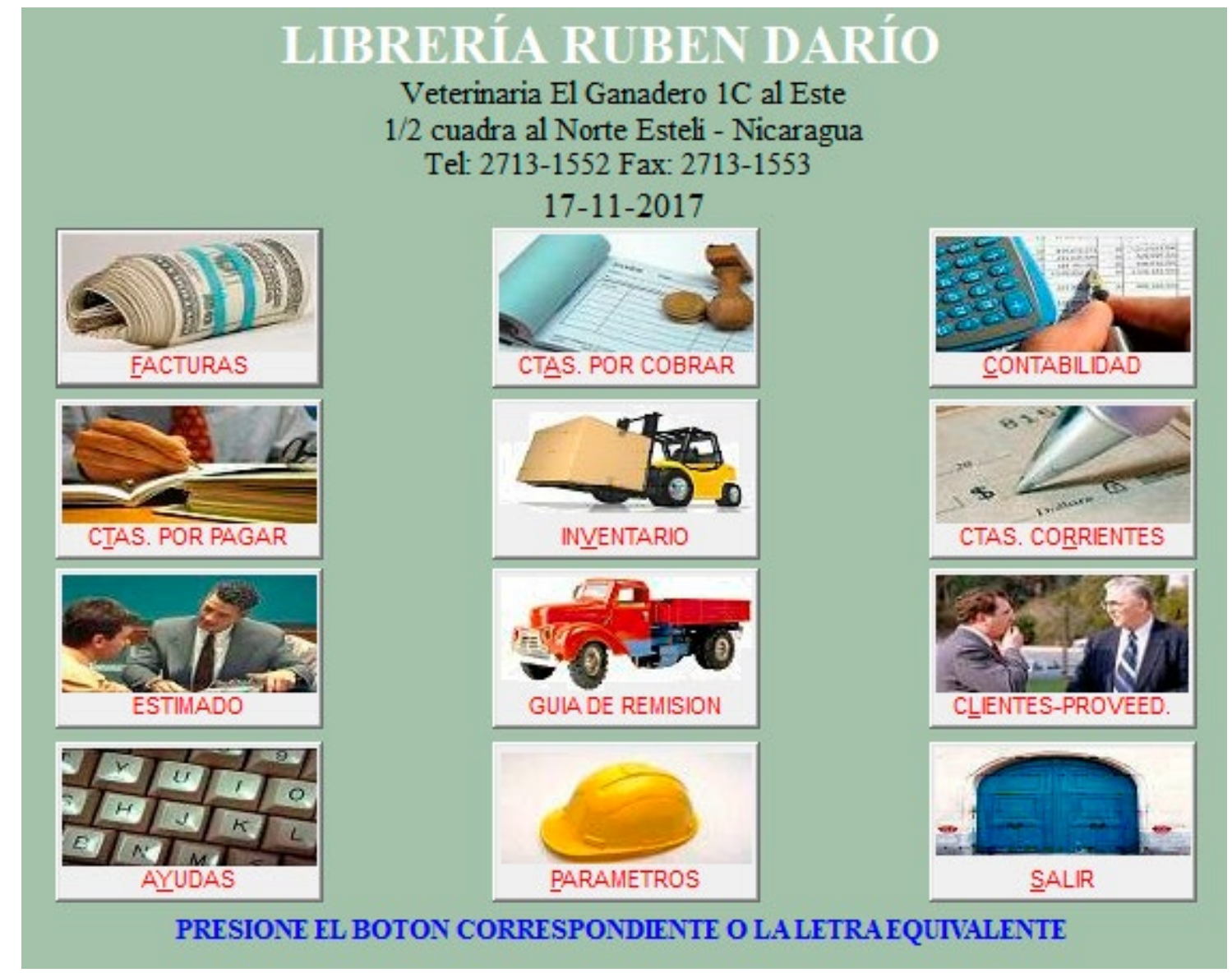

Fuente: Tomado del sistema.

Para usar MONICA en la versión de Windows se necesitan los siguientes requerimientos técnicos:

- Computador con Windows XP (SP3) o versiones siguientes. Recomendamos computadores con Windows 7 o versiones siguientes.

- Computador con 2 GB de memoria RAM. recomendamos almenas 4 GB.

- Disco duro con almenas $10 \mathrm{~GB}$ y velocidad mínima de acceso 7500 RPM. Recomendamos que el disco sea el más rápido posible

- Monitor color con resolución de almenas 1024 x 768 pc.

- Impresora de 80 columnas (Recomendable impresora láser o de inyección "Desjeta").

- En modo de "Punto de Venta" puede usar impresora de recibos.

- Las gavetas que se utilizan para punto de venta son conectadas a impresoras EPSON modelo TMU e impresoras STAR Serie 200 conectadas con cable RJ45. Otros modelos no han sido probados.

- En red de computadoras se recomienda velocidad de transmisión de 100 Bits o superior. 
A los 41 estudiantes se le aplicó una breve encuesta haciendo uso de Google formularios y respondieron 40 estudiantes. Los resultados fueron interesantes, por ejemplo, los estudiantes estaban preocupados por el uso del software contable y sobre todo por el uso de la computadora, lo cual es un aspecto beneficioso. El $80 \%$ de los estudiantes afirmaron que tenían su computadora propia, y un $20 \%$ utiliza una prestada.

El $100 \%$ de los estudiantes contestó que el uso del programa le facilitó el aprendizaje, lo cual es interesante porque la mayoría de ellos aprovecharon el uso, la explicación y los tutoriales que se les proporcionó en el desarrollo del laboratorio.

Sin embargo, un problema que se presentó fueron las condiciones del espacio donde recibían clase los estudiantes, porque los pupitres eran muy pequeños, y solamente había dos conexiones para la energía eléctrica. Pero, poco a poco nos fuimos adecuando a las condiciones. Para las siguientes sesiones se hicieron gestiones para acceder a un espacio más cómodo, y cuando se podía y no estaba ocupado, cumplimos satisfactoriamente la jornada.

Otro recurso que se pudo utilizar fueron los estudiantes monitores. De acuerdo a la historia, fue el educador británico Joseph Lancaster (1778 - 1838) quien ideó un sistema de enseñanza mediante monitores, el cual describió en 1803 en su obra Improvements in Education. Dentro del sistema ideado por el londinense cada alumno avanzaba de acuerdo a su propio ritmo de aprendizaje. Actualmente este modelo didáctico se denomina tutorías- aprender a través de enseñar, se apoya en la idea que se puede lograr un aprendizaje adicional ayudando a otros a aprender, operando así el principio didáctico de aprendizaje entre pares.

Esta modalidad tutorial de monitores se ha desarrollado en otros países, como por ejemplo México, y es parte del programa de servicio social presente en muchas de sus universidades. La inserción temprana de alumnos avanzados en actividades docentes como monitores, ha demostrado que contribuye a reforzar los vínculos con la institución, canalizando tempranas vocaciones hacia la docencia y contribuyendo decididamente a la formación integral de los estudiantes universitarios. Las tutorías entre pares (TEP) se entienden como una forma de atención individualizada que optimiza el aprendizaje, lo interesante es que el uso del blog no necesariamente implica tener una computadora portátil o de escritorio para acceder a ellos.

Monitorear, es la actividad académica que realiza un estudiante con el objeto de colaborar armónicamente con el profesor, bajo su inmediata dirección, en el proceso enseñanza aprendizaje, facilitando a su vez la comunicación profesor-estudiante y promoviendo el interés por una determinada área del saber.

Como una necesidad inminente en el logro de los objetivos se consideró importante apoyar los procesos de enseñanza con estudiantes monitores, se hizo una selección tomando en consideración los siguientes aspectos: ser estudiante activo, buen rendimiento académico, trayectoria académica y conocimiento de área. Se seleccionaron a 10 estudiantes, y a cada uno se les asignó 3 estudiantes, con ellos se trabajaba un día a la semana antes de la sesión de clase, se preparaba la estrategia, se les explicaba lo que haría en clase y ellos apoyaban el proceso.

La experiencia fue de mucho provecho, tanto por el uso de MONICA como por el acompañamiento de los estudiantes monitores, esta metodología se salió del esquema de los papeles, o de archivos en Excel, y los estudiantes se familiarización en el uso de programas computarizados, y con el acompañamiento adecuado, se creó un ambiente de compañerismo, respeto y también destacaron la tolerancia. 


\section{CONCLUSIÓN}

Este semestre se experimentó nuevas técnicas, el uso del programa computarizado de Mónica trajo un resultado provechoso y los más importante el acompañamiento que se hace sentir entre el estudiantado. Se destaca la gran importancia que tuvieron los alumnos monitores en el desarrollo del curso, y en la motivación de los estudiantes, cultivando valores de: respeto, compañerismo, y solidaridad.

Esta experiencia permitió compartir enseñanzas, se aprendió en este camino tanto técnicamente, metodológica y tecnológicamente. Se espera ir mejorando a cada momento, utilizar la tecnología canalizándola provechosamente en todos los ambientes educativos.

Por ello, actualmente se está implementando la estrategia de estudiantes monitores, sobre todo en los grupos numerosos y para atender equitativamente a todos los estudiantes. Esta experiencia demostró que esta estrategia es de mucho provecho.

\section{BIBLIOGRAFÍA}

Aibar, M. (2015). Finanzas personales: Planificación, control y gestión. España: Aula mentor. Caminos, G. B. (2015). Planificación de las finanzas personales. España: Guías Banco Caminos.

Casteñada, C., Pimienta, M. C., \& Jaramillo, P. E. (2008). Usos de TIC en la Educación Superior. Red Iberoamericana de informátiva educativa, 4.

Colombo, P. (2015). Liderando mis Finanzas: Cómo salir de deudas, ahorrar y comenzar a invertir. USA: Kindle Edition.

goconqr.com. (s.f.). goconqr.com. Obtenido de https://www.goconqr.com/es/examtime/blog/glosariode-terminos-educativos/

Sanchis, B. (2014). Idoceo. Obtenido de http://www.idoceo.es/index.php/es/instrucciones/category/15cuaderno-de-notas

significados.com. (2015). www.significados.com. Obtenido de https://www.significados.com/outfit/

Soler Pérez, V. (2008). El uso de las TIC (Tecnologías de la Información y la Comunicación) como herramienta didáctica en la escuela. Contribuciones a las Ciencias Sociales, 1.

Tecnotel. (2010). Manual de uso de Mónica. Estados Unidos. 